\title{
A Search for Rapid Optical Variations in Very Radio- Loud Narrow-Line Seyfert Galaxies
}

\section{J. D. Maune ${ }^{1}$}

Georgia State University

29 Peachtree Center Avenue, Science Annex Suite 400, Atlanta, GA, 30303-4106, USA

E-mail: maune@chara.gsu.edu

\section{H. R. Miller}

Georgia State University

29 Peachtree Center Avenue, Science Annex Suite 400, Atlanta, GA, 30303-4106, USA

E-mail: millerlchara.gsu.edu

\section{J. R. Eggen}

Georgia State University

29 Peachtree Center Avenue, Science Annex Suite 400, Atlanta, GA, 30303-4106, USA

E-mail: eggenechara.gsu.edu

Recent observations appear to indicate that there are fundamental similarities in the properties of the class of objects known as very radio-loud narrow-line Seyfert 1 (RL-NLSy1) galaxies (defined as having a radio loudness $\mathrm{R}>100$ ) and blazars. We report the first results of optical monitoring of the variability of a small sample of RL-NLSy1 galaxies on time scales ranging from hours to days.

Narrow-Line Seyfert 1 Galaxies and Their Place in the Universe (NLS1 Milan (Italy)

April 04-06, 201

\footnotetext{
1 Presenter
} 


\section{Introduction}

Active Galactic Nuclei (AGN) are highly compact and energetic emission sources embedded in the cores of host galaxies. The standard model for AGNs depicts a supermassive black hole exhibiting a pair of collimated relativistic jets that are fueled by an equatorial accretion disk perpendicular to the jets. From this basic model, AGNs can be subdivided into distinct classes, two of which - Seyfert galaxies and blazars - are of relevance to this paper.

Seyfert galaxies are spiral galaxies that are characterized by the presence of strong emission lines and the high luminosity of their nuclei. They are further classified by the width of these emission lines. This paper will largely limit itself to a specific subclass of Seyfert galaxies known as very radio loud narrow-line Seyfert 1 galaxies (RLNLSy1). Blazars are similar objects, save that they have been found to be hosted in elliptical galaxies and are uniquely oriented so that the jet lies along the line of sight to the observer. Due to this orientation and the resultant Doppler boosting, blazars are highly variable on timescales as short as days or even hours.

If the central source in Seyfert galaxies are capable of producing a jet - and the existence of radio-loud Seyfert galaxies suggests that this is possible - then some of them should contain jets which lie along the observer's line of sight. We contend that RLNLSy1s fit this description. One test of this hypothesis is to search for the presence of microvariability, a defining characteristic of blazars. Previously reported observations of J0948+0022 by Liu et al. already claim to have detected extraordinarily large amplitude variations on hourly timescales [1]. We report the results of monitoring the optical variability of this source and several other RLNLSy1s over the course of five nights in an attempt to detect the presence of similarly blazar-like microvariability. If such microvariability was detected, it would support our claim that blazars and RLNLSy1s are fundamentally similar objects.

\section{The Data}

Our sample of objects consisted of a total of 8 RL NLS1s [Table 1] selected from the list contained within Yuan et al [2]. Observations were made using the PRISM instrument of the $1.8 \mathrm{~m}$ Perkins telescope at the Lowell observatory in Flagstaff, AZ. All data were collected in the standard R band filter on the nights of March 4-7, 2011. In-field standard stars were chosen based on images obtained from the SDSS DR8. As these comparison stars remain uncalibrated as of the publication of this paper, differential magnitudes are reported. To ensure that the selected comparisons are themselves unvarying, check-check light curves are also demonstrated.

\section{Conclusions}

Of the eight RLNLSy1s we monitored, microvariability was detected in two: J0948+0022 and J1305+5116, with the former being the stronger detection [Fig. 1]. However, 

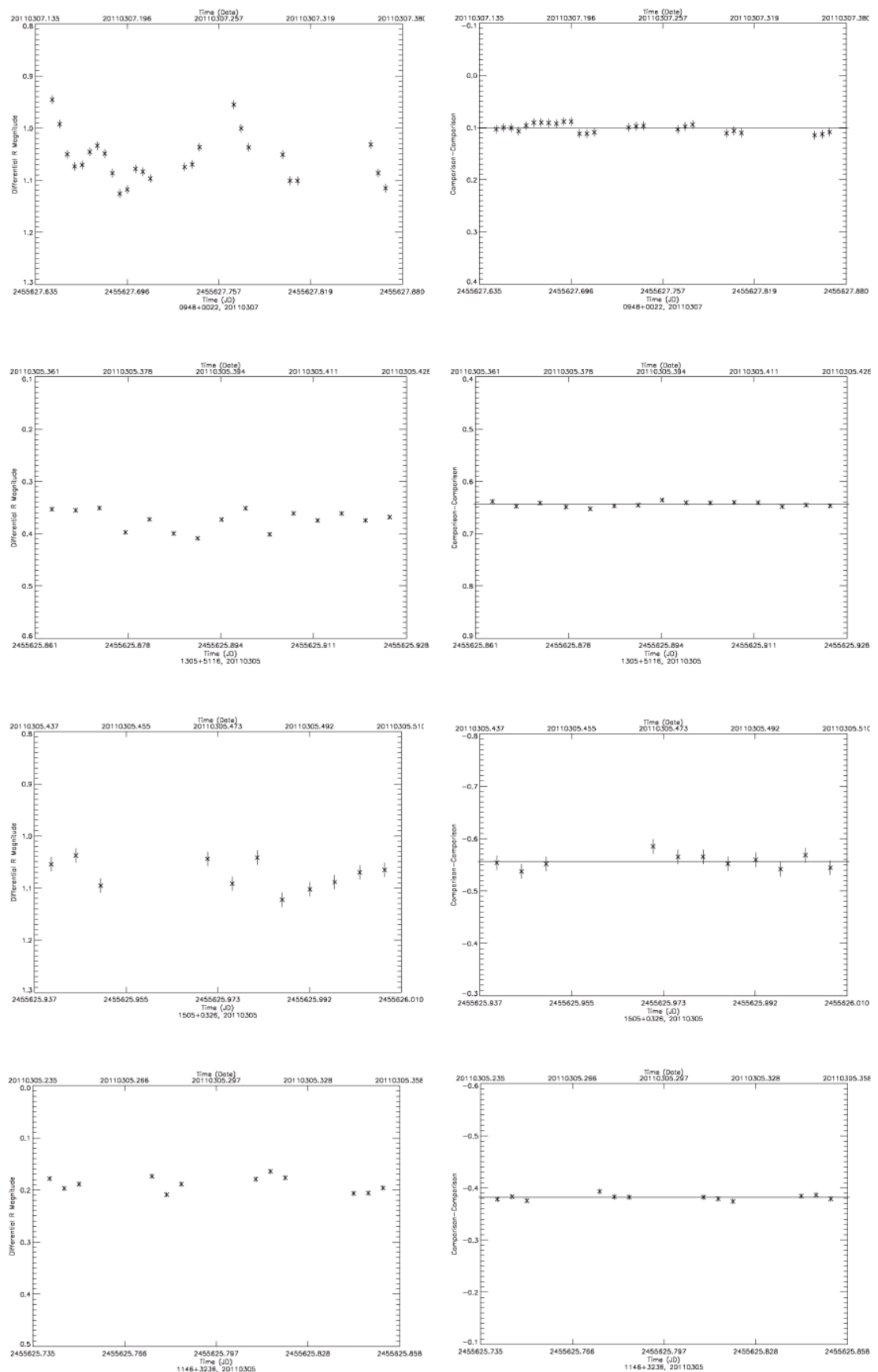

Fig. 1: Light curves for selected sources (left) and accompanying comparison star - comparison star information (right).

Top: J0948+0022. This object demonstrated the clearest example of blazar-like microvariability. Middle top: J1305+5116. Observed microvariability is minor, but clear trends can still be seen.

Middle bottom: 1505+0326. A tentative detection, but further observations will be required.

Bottom: J1146+3236. A null result. Some scatter is seen, but no trends are detected. 


\begin{tabular}{|l|l|l|}
\hline J0948+0022 & 094857.3175 & +002225.511 \\
\hline J1146+3236 & 114657.0655 & +323658.164 \\
\hline J1305+5116 & 130522.7562 & +511640.188 \\
\hline J1435+3131 & 143605.3123 & +312927.639 \\
\hline J1443+4725 & 144318.5579 & +472556.663 \\
\hline J1505+0326 & 150502.6660 & +032622.910 \\
\hline J1633+4718 & 163319.2590 & +471656.770 \\
\hline J1644+2619 & 164442.5340 & +261913.305 \\
\hline
\end{tabular}

Table 1: objects of interest (RA and Dec

taken from Simbad, Epoch 2000)

significant microvariability (with an amplitude of $0.18 \mathrm{mag}$ ), was detected on only one of the four nights J0948+0022 was observed. This is in sharp contrast to the results of Liu et al. which reported microvariations $\sim 0.5 \mathrm{mag}$ for all seven nights they monitored this source. Three more of our objects (J1505+0326, J1633+4718, and J1644+2619) appear to demonstrate signs of microvariability, but this cannot currently be confirmed due to the quality of the available data. The remaining three objects $(\mathrm{J} 1146+3236, \mathrm{~J} 1436+3131$, and $\mathrm{J} 1443+4725)$ showed no signs of microvariability. It should be noted, however, that even known blazars will occasionally undergo intervals in which they enter a quiescent state; further, in a study of x-ray selected BL Lac objects it was observed that on any given night, only 34\% of all XBLs can be expected to demonstrate microvariability [3]. It would therefore not be particularly surprising if later observations of the inactive RLNLSy1s showed increased variability given the results of the remainder of the sample.

Having demonstrated that radio-loud NLSy1s are variable, it is logical to ask if their radio-quiet counterparts are similarly capable. A study by Miller et al. found that this was true for one of five radio-quiet NLSy1s under observation, though there was some ambiguity to their null detections in a manner similar to our own data [4]. This variability may be associated with events occurring within the accretion disk, and demonstrates that detection of microvariability alone cannot be used to determine a blazar-like orientation. Instead, a combination of factors such as radio-loudness and polarization must be examined in addition to variability in order to make a determination. A forthcoming paper by Eggen et al. will be exploring the issue of polarization, to be released alongside this one in PoS [5].

Regardless, the detection of blazar-like microvariability in at least some RLNLSy1s supports the claim that these two classes of objects share similar characteristics.

\section{References}

[1] Liu et al., Violent Intranight Optical Variability of a Radio- Loud Narrow-Line Seyfert 1 Galaxy: SDSS J094857.3+002225, ApJ Letters, 715:L113-L116, 2010

[2] W. Yuan et al., A Population of Radio-Loud Narrow-Line Seyfert 1 Galaxies with Blazar-Like Properties, ApJ 685:801-827, 2008

[3] A. Cambell, Variability Characteristics of X-Ray Selected Blazars, PhD Dissertation, Georgia State University, 2004 
[4] H. Miller et al., Evidence of Rapid Optical Variability in Selected Narrow-Line Seyfert 1 Galaxies, New Astronomy Reviews, vol. 44, issues 7-9, pages 539-541, 2000

[5] J. Eggen et al., Optical Polarization and FERMI Observations of Radio-Loud NLSyl Galaxies, forthcoming 2011 